\title{
Expression of MMPs is dependent on the activity of mitogen-activated protein kinase in chondrosarcoma
}

\author{
MIN YAO $^{1,2}$, XIAOMEI WANG ${ }^{1}$, YUFENG ZHAO $^{1}$, XIAOMENG WANG $^{1}$ and FENG GAO ${ }^{1}$ \\ ${ }^{1}$ Department of Pathology, The Third Hospital of Hebei Medical University, Shijiazhuang, Hebei 050051; \\ ${ }^{2}$ Department of Biochemistry, Hebei Medical University, Shijiazhuang, Hebei 050017, P.R. China
}

Received November 9, 2015; Accepted November 10, 2016

DOI: $10.3892 / \mathrm{mmr} .2016 .6077$

\begin{abstract}
Matrix metalloproteinases (MMPs) and tissue inhibitors of matrix metalloproteinases (TIMPs) serve an important role in chondrosarcoma. The present study investigated whether the expression of MMPs was dependent on the activity of mitogen-activated protein kinase (MAPK) in chondrosarcoma. Surgical pathological specimens were collected to detect MMP-1, MMP-13, TIMP-1, type II collagen and phosphorylated MAPK levels in normal cartilage, enchondroma and chondrosarcoma tissues. The expression of MMP-1, MMP-13, TIMP-1 and type II collagen was investigated utilizing MAPK inhibitors in chondrosarcoma cells. It was noted that the expression levels of MMP-1, MMP-13 and TIMP-1 were increased in chondrosarcoma with the activity of MAPK. After chondrosarcoma cells were pretreated with MAPK inhibitors, the levels of MMP-1, MMP-13 and TIMP-1 were inhibited. Furthermore, MMP-1 and MMP-13 are essential in regulating the degradation of type II collagen and decomposing cartilage matrix major. The high expression levels of MMP-1 and MMP-13 in chondrosarcoma expedite the invasion by chondrosarcoma cells and their expression can be depressed by MAPK inhibitors.
\end{abstract}

\section{Introduction}

Cartilage tumors are a common type of bone tumor and the second most prevalent primary skeletal tumors $(1,2)$. The main cartilage tumors include osteochondroma, enchondroma, chondroblastoma, chondromyxoid fibroma and chondrosarcoma. Chondrosarcoma is a malignant cartilage tumor, highly resistant to conventional chemotherapy and radiotherapy, and commonly surgical resection is the only effective treatment $(3,4)$. Due to

Correspondence to: Dr Feng Gao, Department of Pathology, The Third Hospital of Hebei Medical University, 139 Ziqiang Road, Shijizhuang, Hebei 050051, P.R. China

E-mail: alan846829@163.com

Key words: chondrosarcoma, matrix metalloproteinases, tissue inhibitors of matrix metalloproteinases, mitogen-activated protein kinase the absence of an effective adjuvant therapy, this mesenchymal malignancy has a poor prognosis. The invasion and metastasis mechanism of malignant tumors is a complex process, which includes decomposing the extracellular matrix (ECM), degrading the basal membrane and invading lymphatic and blood vessels. Chondrosarcoma invades and metastasizes through this mechanism (5) and inhibiting the ability of chondrosarcoma cells to decompose ECM can prohibit the relapse and metastasis of chondrosarcoma.

Matrix metalloproteinases (MMPs) and tissue inhibitors of matrix metalloproteinases (TIMPs) serve a significant role in decomposing the ECM. Previous studies have reported that MMPs decompose all components of the ECM $(6,7)$. According to their structure and substrate specificity they can be divided into subgroups of collagenases, gelatinases, stromelysins, membrane-type MMPs and other MMPs. MMP-1 and MMP-13 are collagenases, whose biochemical characterization is to decompose type I, II and III collagen (8). TIMP-1 is the tissue inhibitor of MMP-1 and MMP-13. It has been reported that MMPs were highly expressed in some tumor tissues, such as tumors of the digestive system, the kidneys, ovaries and lungs (9-11). These tumor cells likely enhance their invasive capability through MMPs to decompose ECM. It has been demonstrated that MMP-1 and MMP-13 are highly expressed in chondrosarcoma (12) and these high expression levels contribute to the decomposition of type II collagen.

The mitogen-activated protein kinase (MAPK) pathways are focal points for diverse extracellular stimuli and regulate the activities of kinases or transcription factors downstream, thereby influencing gene expression and cellular responses (13). Three of the most well-known MAPK pathways have been characterized in detail: Extracellular signal-regulated kinase 1/2 (ERK1/2), c-jun N-terminal kinases (JNK) and the p38 pathway (14). MAPK pathways regulate a number of transcription factors such as activator protein-1 and nuclear factor- $\kappa \mathrm{B}$, which act independently or in concert to regulate numerous genes involved in the regulation of urinary plasminogen activator (u-PA) and MMP expression (15). Previous studies have demonstrated that MAPK pathways regulated MMP expression in breast carcinoma, non-small cell lung carcinoma and liver cancer, however the association between MAPK pathways and MMPs in chondrosarcoma remains unclear (16-18). In the present study, the expression levels of collagenases (MMP-1 and MMP-13) and MAPK pathways in 
chondrosarcoma specimens and chondrosarcoma cells were compared on order to investigate whether MAPK-dependent induction of MMP expression can enhance the invasive capability of chondrosarcoma by decomposing cartilage matrix. The present study explored the relative associations between MAPK pathways and MMPs in chondrosarcoma, and provides a theoretical basis for curing chondrosarcoma with MAPK inhibitors.

\section{Materials and methods}

Patients and specimen preparation. A total of 79 histologically examined surgical specimens were obtained from the patients of the Third Hospital of Hebei Medical University (Shijiazhuang, China) between 2012 and 2014, after approval by the ethics committee of the Third Hospital of Hebei Medical University. All specimens were divided into three groups according to degree of malignancy: Normal cartilage tissue $(n=17)$, enchondroma tissue $(n=25)$ and chondrosarcoma tissue $(n=37)$. The tissue specimens were obtained from surgical and pathological records at the hospital. Enchondroma tissue was obtained from 15 male and 10 female patients (age, 34-62 years; mean age, 48 years). Chondrosarcoma tissue was obtained from 21 males and 16 females patients (age, 38-72 years; mean age of 56 years). Specimens of normal cartilage tissue were obtained from knee joints of patients. Fresh pathological specimens were stored in an ultra-low temperature freezer before western blotting and reverse transcription-quantitative polymerase chain reaction (RT-qPCR). All specimens were fixed in $4 \%$ paraformaldehyde in $0.01 \mathrm{~mol} / 1$ phosphate-buffered saline (PBS) before embedding in paraffin wax.

Cell culture. The human chondrosarcoma cell line (SW1353) was obtained from the American Type Culture Collection (Manassas, VA, USA). The cells were cultured in Dulbecco's modified Eagle's medium and a-modified Eagle's medium supplemented with $10 \%$ fetal bovine serum and maintained at $37^{\circ} \mathrm{C}$ in a humidified atmosphere of $5 \% \mathrm{CO}_{2}$. The cells were incubated for $48 \mathrm{~h}$ and were pretreated with the ERK1/2 inhibitor, U0126 (10 $\mu \mathrm{mol} / \mathrm{l}$; Sigma-Aldrich; Merck Millipore, Darmstadt, Germany), JNK inhibitor, SP600125 (20 $\mu \mathrm{mol} / \mathrm{l}$; Sigma-Aldrich; Merck Millipore) and p38 inhibitor, SB203580 (10 $\mu \mathrm{mol} / \mathrm{l}$; Sigma-Aldrich; Merck Millipore) (19). All the compounds were dissolved in dimethyl sulfoxide $\left(\mathrm{Me}_{2} \mathrm{SO}\right)$.

Immunohistochemistry. Paraffin wax-embedded renal tissue sections $(4 \mu \mathrm{m})$ were dewaxed with xylene and rehydrated in graded ethanol solutions. Endogenous horseradish peroxidase activity was blocked by pretreatment with $3 \% \mathrm{H}_{2} \mathrm{O}_{2}$ for $10 \mathrm{~min}$ at room temperature. Antigen recovery was performed using a microwave. To block nonspecific binding, the sections were incubated at $37^{\circ} \mathrm{C}$ for $30 \mathrm{~min}$ in PBS containing $10 \%$ goat serum. Finally, the sections were incubated with rabbit polyclonal antibodies against MMP-1 (catalog no. sc-8834; 1:50; Santa Cruz Biotechnology, Inc., Dallas, TX, USA), MMP-13 (catalog no. sc-30073; 1:100; Santa Cruz Biotechnology, Inc.), TIMP-1 (catalog no. bs-0415R; 1:50; Bioss Biotechnology, Beijing, China) and type II collagen (catalog no. sc-28887; 1:50 dilution; Santa Cruz Biotechnology, Inc.), overnight at $4^{\circ} \mathrm{C}$. On the following day, after incubation with the PV-9000 Polymer
Detection System (OriGene Technologies, Inc., Beijing, China), the sections were stained with 3,3-diaminobenzidine and counterstained with hematoxylin. Negative controls were obtained by replacing the primary antibody with PBS.

Western blot analysis. Pathological specimens and SW1353 cells were lysed and protein concentrations were measured by Coomassie brilliant blue assay. Proteins were loaded and separated on a $10 \%$ SDS-polyacrylamide gel and then transferred to a polyvinylidene difluoride membrane. The membrane was blocked with $5 \%$ dried milk and incubated overnight at $4{ }^{\circ} \mathrm{C}$ with rabbit anti-MMP-1 (1:200), MMP-13 (1:500) TIMP-1 (1:200), type II collagen (1:200) phosphorylated (p)-ERK1/2 (catalog no. sc-101760; 1:100; Santa Cruz Biotechnology, Inc.), ERK1/2 (catalog no. 4695; 1:200; Cell Signaling Technology, Inc., Danvers, MA, USA), p-JNK (catalog no. sc-135642; 1:100; Santa Cruz Biotechnology, Inc.), JNK (catalog no. 9252; 1:200; Cell Signaling Technology, Inc.), p-p38 (catalog no. 4511; 1:200; Cell Signaling Technology, Inc.), p38 (catalog no. sc-7149; 1:100; Santa Cruz Biotechnology, Inc.) and $\beta$-actin (catalog no. bs-0061R; 1:1,000; Bioss Biotechnology). After washing, the membrane was incubated with a horseradish peroxidase-conjugated chemiluminescent secondary antibody (catalog no. NA932; 1:1,000; GE Healthcare Bio-Sciences, Pittsburgh, PA, USA). Proteins were quantified following acquisition and analysis of the image using the LabWorks software, version 4.5, with the UVP Imaging Station, (UVP, Inc., Upland, CA, USA). Protein expression was quantified by comparison with the internal control $\beta$-actin.

$R T-q P C R$. Total RNA was collected using TRIzol Reagent (Sigma-Aldrich; Merck Millipore) following the manufacturer's protocol. RNA (1 $\mu \mathrm{g})$ was reverse transcribed using the oligo (dT) primer in the presence of avian myeloblastosis virus reverse transcriptase to produce cDNA. Successful cDNA production was verified by PCR using the PCR Master Mix (Eppendorf North America, Westbury, NY, USA). The primers were supplied as a pre-optimized single tube primer/probe Gene Expression Assay (Applied Biosystems; Thermo Fisher Scientific, Inc., Waltham, MA, USA). The two oligonucleotide primers were: Glyceraldehyde-3-phosphate dehydrogenase (GAPDH), forward 5'-ACCACAGTCCATGCCATCAC-3' and reverse 5'-TCCACCACCCTGTTGCTGTA-3'; MMP-1, forward 5'-AAGCCAGATGCTGAAACCCTG-3' and reverse 5'-GACCCTTGGAGACTTTGGTGAAT-3'; MMP-13, forward 5'-TTGACCACTCCAAGG ACCCAG-3' and reverse 5'-GAGGATGCAGACGCCAGAAGA-3'; TIMP-1, forward 5'-ACAGGTTTCCGGTTGG-3' and reverse 5'-CAGGCA GGCAAAGTGAT-3'. Each PCR cycle was conducted for 33 cycles of $30 \mathrm{sec}$ at $94^{\circ} \mathrm{C}, 30 \mathrm{sec}$ at $55^{\circ} \mathrm{C}$ and $1 \mathrm{~min}$ at $68^{\circ} \mathrm{C}$. The PCR products were then separated electrophoretically in a $2 \%$ agarose DNA gel and stained with ethidium bromide; PCR reactions were set up with Taqman Universal PCR Master Mix from Applied Biosystems (Thermo Fisher Scientific, Inc., Waltham, MA, USA). cDNA was amplified on the $7900 \mathrm{HT}$ Sequence Detection System (Applied Biosystems; Thermo Fisher Scientific, Inc.) at default thermal cycling conditions: $2 \mathrm{~min}$ at $50^{\circ} \mathrm{C}, 10 \mathrm{~min}$ at $95^{\circ} \mathrm{C}$ for enzyme activation and then 40 cycles of $15 \mathrm{sec}$ at $95^{\circ} \mathrm{C}$ for denaturation and $1 \mathrm{~min}$ at $60^{\circ} \mathrm{C}$ for annealing and extension. Results were analyzed using the 


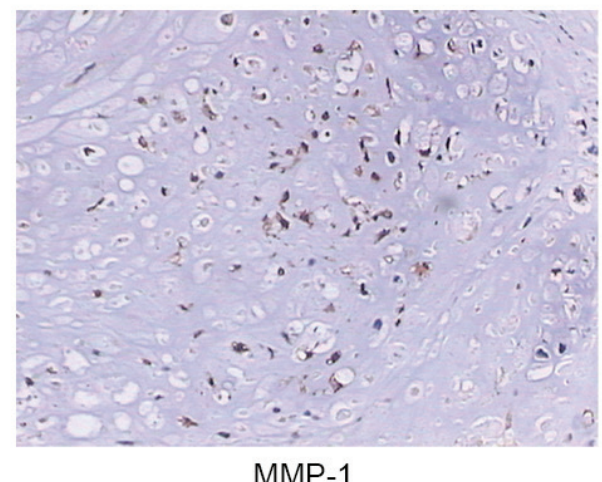

MMP-1

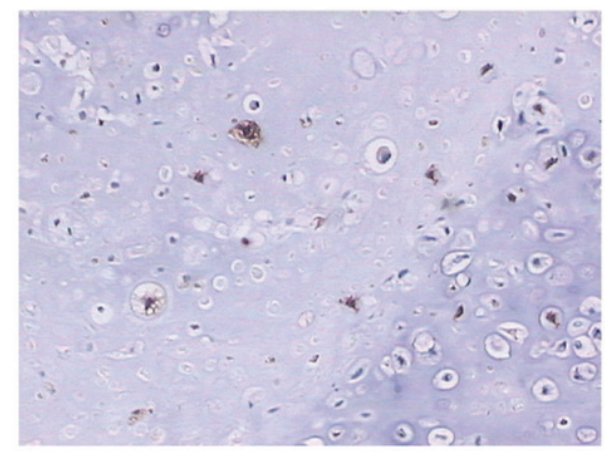

TIMP-1

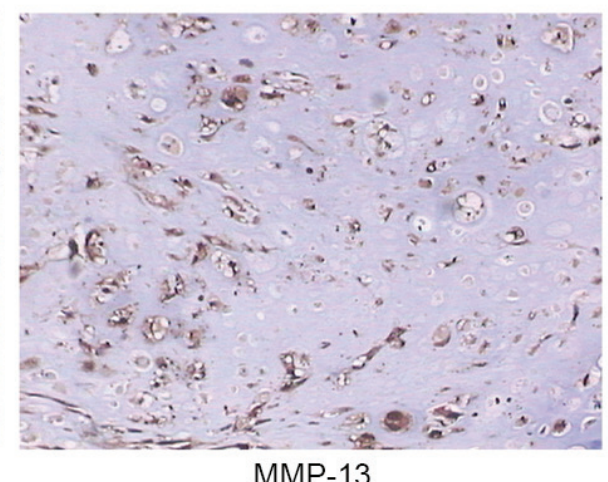

MMP-13

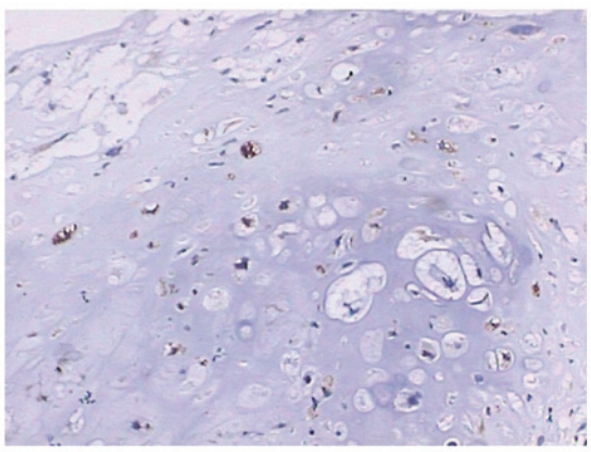

Type II collagen

Figure 1. Protein expression of MMP-1, MMP-13, TIMP-1 and type II collagen in chondrosarcoma by immunohistochemistry (magnification, x100). MMP, matrix metalloproteinase; TIMP, tissue inhibitor of matrix metalloproteinase.

relative standard curve method of analysis/ $\triangle C q$ method of analysis (20).

Statistical analysis. Data were expressed as the mean \pm standard deviation and were analyzed using one-way analysis of variance with Bonferroni's post hoc test by SPSS software version 13.0 (SPSS, Inc., Chicago, IL, USA). In all cases, $\mathrm{P}<0.05$ was considered to indicate a statistically significant difference.

\section{Results}

Expression of MMP-1, MMP-13, TIMP-1 and type II collagen in pathological specimens. Immunohistochemical results (Fig. 1) demonstrated the protein expression of MMP-1, MMP-13, TIMP-1 and type II collagen in the cytoplasm of chondrosarcoma cells. Their protein levels were evaluated in human normal cartilage, enchondroma, and chondrosarcoma tissues by western blotting (Fig. 2A and B). Analysis of protein levels indicated a significant increase of MMP-1 and TIMP-1 protein in the enchondroma compared with cartilage tissue $(\mathrm{P}<0.01)$. No change of MMP-13 and type II collagen protein expression was identified in enchondroma and cartilage tissues ( $\mathrm{P}>0.05)$. The protein expression of MMP-1, MMP-13 and TIMP-1 in chondrosarcoma was significantly higher than that in enchondroma $(\mathrm{P}<0.01)$. Chondrosarcoma tissues exhibited a decreased type II collagen level compared with that of enchondroma $(\mathrm{P}<0.01)$. RT-qPCR demonstrated similar changes of MMP-1, MMP-13, TIMP-1 and type II collagen mRNA in normal cartilage, enchondroma and chondrosarcoma tissues $(\mathrm{P}<0.01$; Fig. 2C).
Expression of MAPK in chondrosarcoma specimens. The activation of ERK1/2, JNK and p38 was detected with antibodies against the phosphorylated forms of the kinases by western blotting (Fig. 3). The protein levels of p-ERK1/2 and p-p38 in chondrosarcoma were significantly increased compared with those in enchondroma and cartilage tissues $(\mathrm{P}<0.01)$. The difference in protein expression between enchondroma and cartilage tissues was not observed as significant $(\mathrm{P}>0.05)$. No activation of JNK was detected in pathological specimens $(\mathrm{P}>0.05)$.

Activation of ERK1/2 and p38 in human chondrosarcoma cells. Activation of ERK1/2 and p38 was identified in chondrosarcoma pathological specimens. Western blot analysis (Fig. 4) indicated that p-ERK1/2 and p-p38 had higher expression levels in SW1353 cells. With an ERK1/2 inhibitor (U0126) and a p38 inhibitor (SB203580), activation of ERK1/2 and p38 was significantly inhibited $(\mathrm{P}<0.01)$. Activation of JNK was not detected in SW1353 cells, whether pretreated by JNK inhibitor (SP600125) or not (P>0.05).

Expression of MMP-1, MMP-13, TIMP-1 and type II collagen in human chondrosarcoma cells is dependent on MAPK activity. The present study identified higher expression levels of MMP-1, MMP-13 and TIMP-1 in clinical chondrosarcoma specimens together with lower levels of type II collagen, and with the activation of ERK1/2 and p38. Subsequently, the specific roles of ERK1/2 and p38 pathways in the regulation of the expression of MMP-1, MMP-13, TIMP-1 and type II collagen were investigated by utilizing two selective chemical inhibitors; U0126 to block 


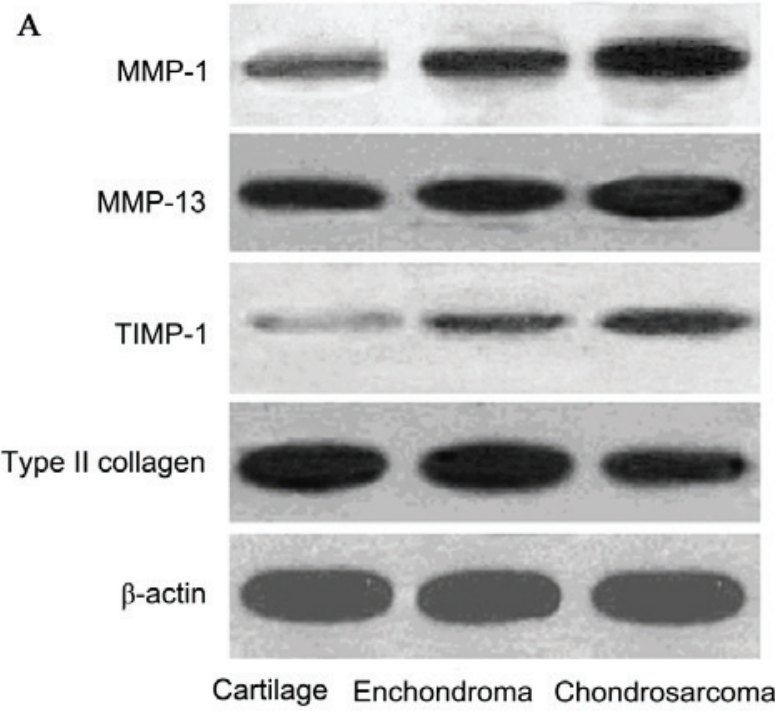

B

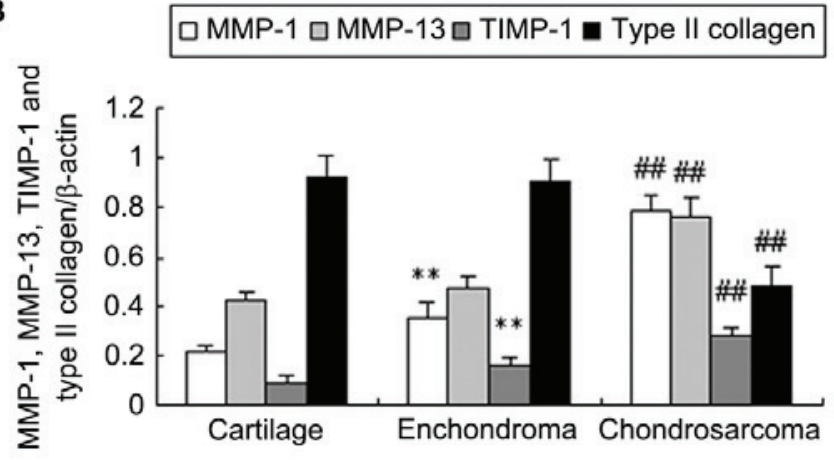

C

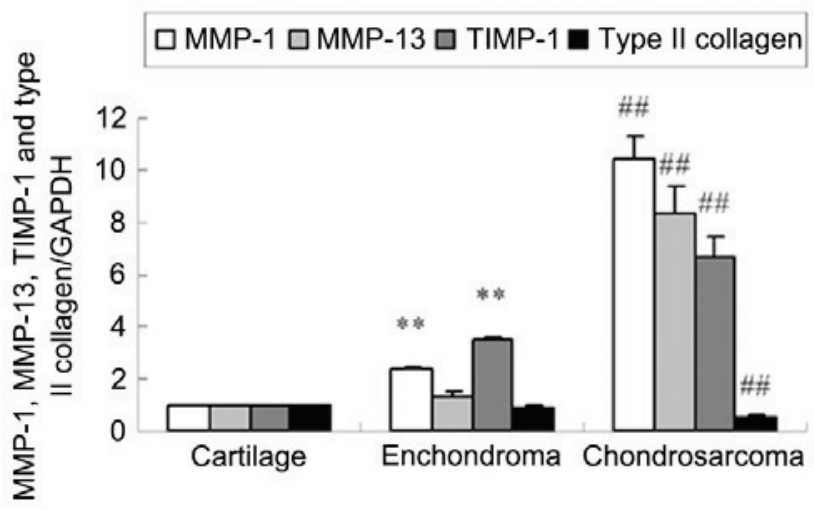

Figure 2. Expression of MMP-1, MMP-13, TIMP-1 and type II collagen in normal cartilage, enchondroma and chondrosarcoma tissues. (A and B) The protein expression of MMP-1, MMP-13, TIMP-1 and type II collagen were analyzed using western blotting. (C) The mRNA levels of MMP-1, MMP-13, TIMP-1 and type II collagen were detected using reverse transcription-quantitative polymerase chain reaction. Data are expressed as the mean \pm standard deviation. ${ }^{* *} \mathrm{P}<0.01$ vs. cartilage tissue. ${ }^{\# \#} \mathrm{P}<0.01$ vs. enchondroma tissue. MMP, matrix metalloproteinase; TIMP, tissue inhibitor of matrix metalloproteinase.

A

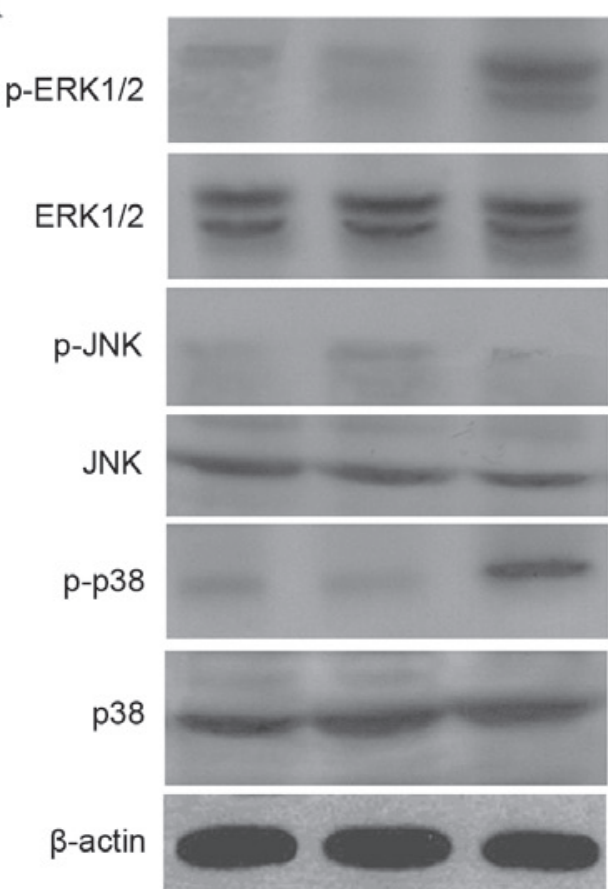

B

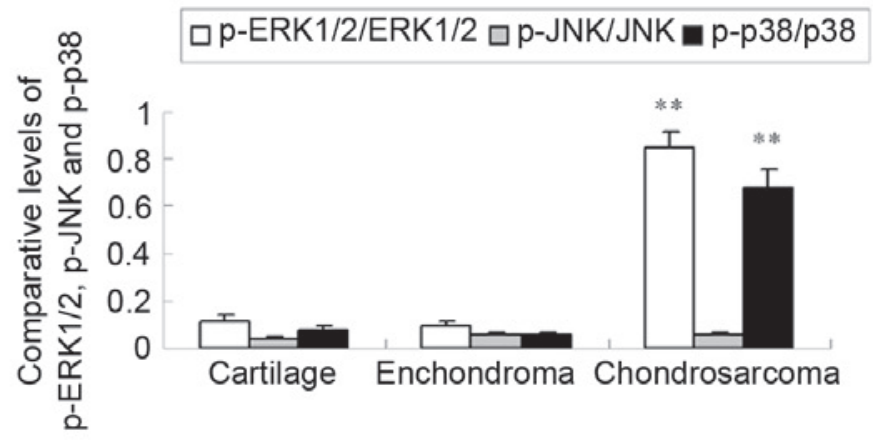

Cartilage Enchondroma Chondroblastoma

Figure 3. Expression of mitogen-activated protein kinase protein levels in normal cartilage, enchondroma and chondrosarcoma tissues. The protein expression of p-ERK1/2, ERK1/2, p-JNK, JNK, p-p38 and p38 was analyzed using (A) western blotting with (B) the quantification presented. Data are expressed as the mean \pm standard deviation. ${ }^{* *} \mathrm{P}<0.01$ vs. cartilage tissue. p-, phosphorylated; ERK1/2 extracellular signal-regulated kinase 1/2; JNK, c-jun N-terminal kinase. 
A

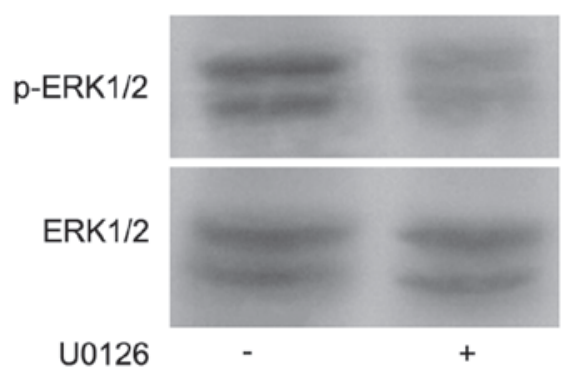

C

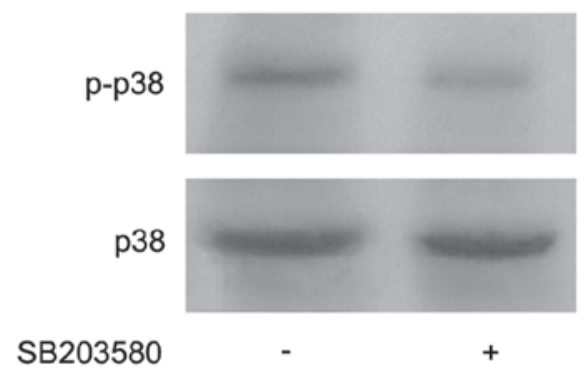

E

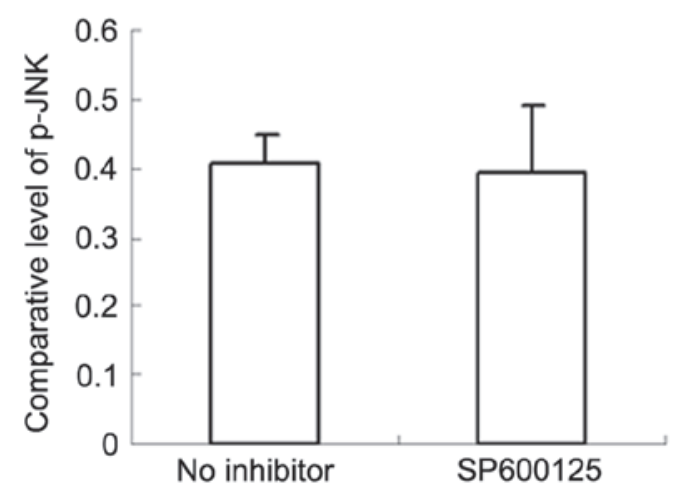

B

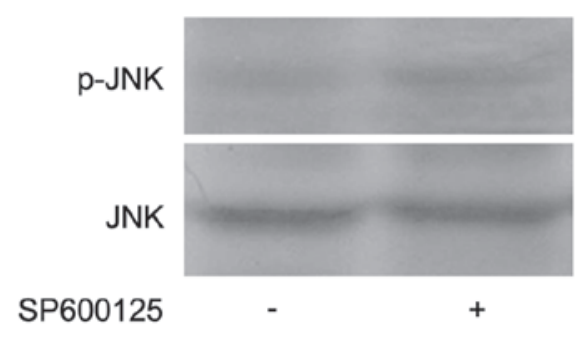

D

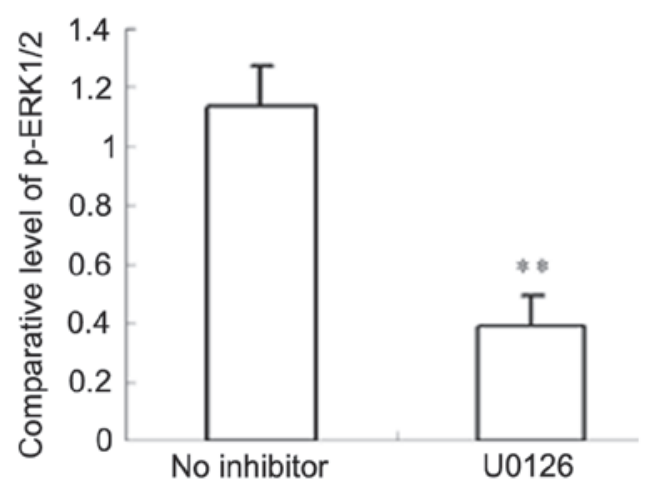

F

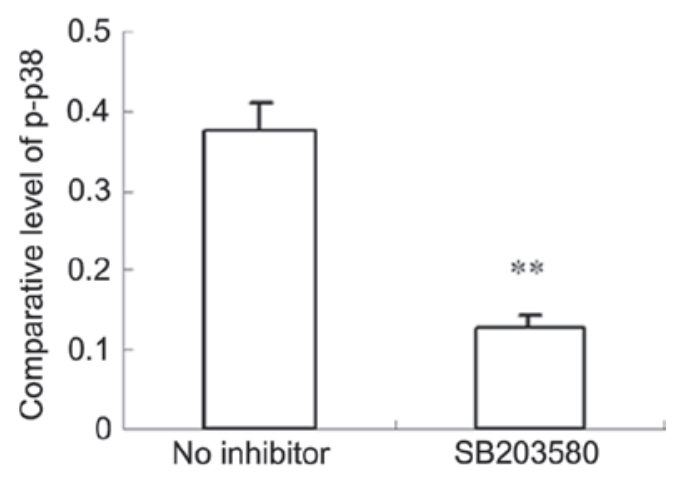

Figure 4. Expression of MAPK protein levels in SW1353 cells treated by MAPK inhibitors. The protein expression levels of (A) p-ERK1/2 and ERK1/2, (B) p-JNK and JNK and (C) p-p38 and p38 were analyzed using western blotting with respective quantification presented in (D). (E and F) Data are expressed as the mean \pm standard deviation. ${ }^{* *} \mathrm{P}<0.01$ vs. no inhibitor. MAPK, mitogen-activated protein kinase; p-, phosphorylated; ERK1/2, extracellular signal-regulated kinase 1/2; JNK, c-jun N-terminal kinase.

the ERK1/2 pathway and SB203580 to inhibit p38 activity. Western blot analysis (Fig. 5A and B) detected that ERK1/2 and 38 inhibitors decreased MMP-1, MMP-13 and TIMP-1 protein expression $(\mathrm{P}<0.01)$. However, ERK1/2 and $\mathrm{p} 38$ inhibitors increased type II collagen protein level $(\mathrm{P}<0.01)$. RT-qPCR (Fig. 5C) indicated similar results for MMP-1, MMP-13, TIMP-1 and type II collagen mRNA expression $(\mathrm{P}<0.05$ or $\mathrm{P}<0.01)$.

\section{Discussion}

Unlike osteosarcoma and Ewing's sarcoma, for which clear increases in long-term survival have been observed as the result of the use of systemic chemotherapy, chondrosarcoma has a poor prognosis due to the absence of an effective adjuvant therapy $(21,22)$. Therefore, it is important to develop an effective adjuvant therapy to prevent chondrosarcoma metastasis. Enzymatic degradation of ECM is one of the crucial steps in tumor invasion and metastasis. MMPs are a family of structurally associated zinc-dependent neutral endopeptidases that are collectively capable of degrading essentially all ECM components, and they are suggested to serve an important role in ECM degradation in tumor invasion. Each type of MMP degrades a specific component of the ECM $(23,24)$.

TIMPs are a family of multifunctional agents, including TIMP-1, TIMP-2, TIMP-3 and TIMP-4, which are a group of proteinase inhibitors secreted by in vivo cells that can produce a marked effect on various tumor types by 
A

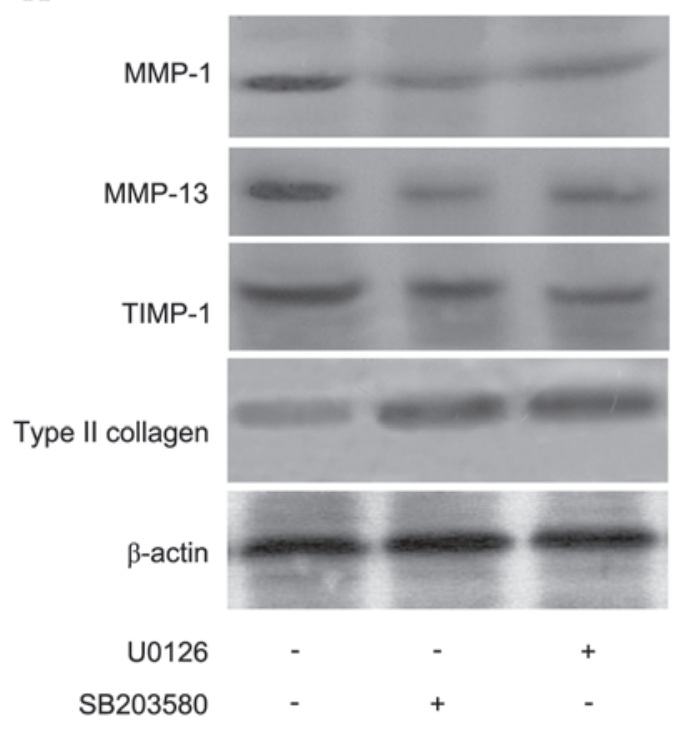

B

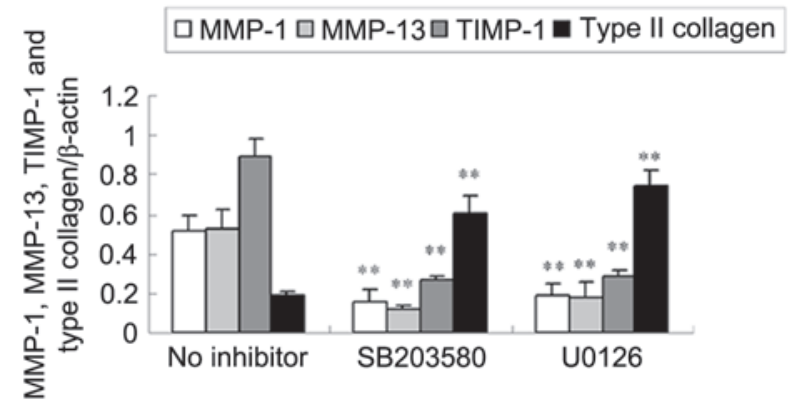

\section{$\mathrm{C}$}

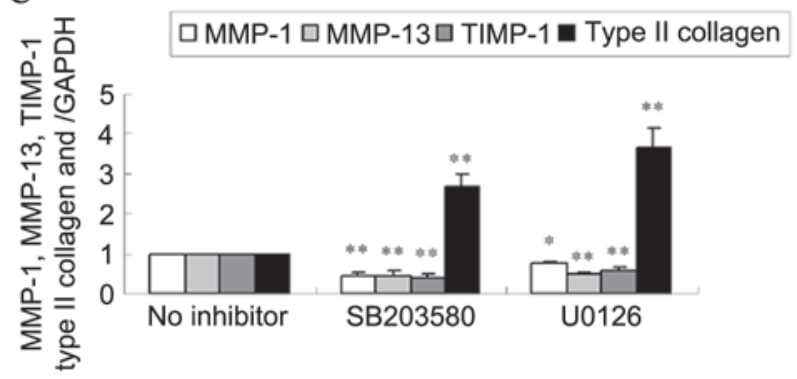

Figure 5. Expression of MMP-1, MMP-13, TIMP-1 and type II collagen in SW1353 cells treated by MAPK inhibitors. (A) The protein expression of MMP-1, MMP-13, TIMP-1 and type II collagen was analyzed using western blotting and (B) the quantification was presented. (C) The mRNA levels of MMP-1, MMP-13, TIMP-1 and type II collagen were detected using reverse transcription-quantitative polymerase chain reaction. Data are expressed as the mean \pm standard deviation. ${ }^{*} \mathrm{P}<0.05,{ }^{* * *} \mathrm{P}<0.01$ vs. no inhibitor. MMP, matrix metalloproteinase; TIMP, tissue inhibitor of matrix metalloproteinase; MAPK, mitogen-activated protein kinase.

inhibiting MMPs $(25,26)$. Previous studies have demonstrated the different expression of MMPs in various types of tumor tissue $(27,28)$. The results of the present study demonstrated that enchondroma had a higher expression level of MMP-1 compared with that of normal cartilage tissue, which suggests that in benign cartilage tumors, certain MMPs have already been activated. Furthermore, chondrosarcoma tissue and cells had significantly higher expression levels of MMP-1 and MMP-13. Chondrosarcoma cells degrade cartilage matrix that contains type II collagen through the increasing expression of MMP-1 and MMP-13, which can enhance the invasion and metastasis of chondrosarcoma cells.

TIMP-1 is the specific inhibitor of MMP-1 and MMP-13, and its higher expression level can depress their activity. It is important to maintain the relative level of MMP-1, MMP-13 and TIMP-1 to control the proper degradation of ECM. Certain in vitro experiments have confirmed that TIMP-1 in malignant tumor cells can depress the expression activity of MMPs and tumor invasion $(29,30)$. The expression of type II collagen, the major component in the structure of cartilage tissue, in chondrosarcoma was decreased, which may be associated with the degradation of MMPs.

ERK $1 / 2$ is commonly implicated in cell proliferation, differentiation and survival (31), whereas p38 is preferentially activated by inflammatory cytokines, cellular stresses, withdrawal of growth factors and proapoptotic stimuli (11). Evidence for the role of MAPKs in malignant transformation was initially provided by the observation that constant activation of ERK1/2 by constitutively active Raf or MAPK/ERK kinase 1/2 results in transformation of the glandular epithelium (32). Furthermore, previous studies have demonstrated that the ERK1/2 pathway is activated in renal and breast carcinoma in vivo $(33,34)$, providing further evidence that the constant activation of ERK1/2 is involved in the malignant transformation of cells. However, the consequences of ERK1/2 activation are cell specific, as demonstrated by the observation that constant activation of the ERK1/2 cascade by Raf can cause growth arrest in small cell lung carcinoma cells (35). An additional study demonstrated that p38 elevated the u-PA expression level and promoted breast carcinoma invasion (36). Miao et al (37) demonstrated that $\mathrm{p} 38$ can encourage colon carcinoma cell line proliferation, further influencing MMPs levels and ECM degradation. The results of the present study also demonstrated activation of ERK1/2 and p38 in chondrosarcoma tissue and cells. MMP-1, MMP-13 and TIMP-1 overexpression were inhibited by a selective ERK1/2 inhibitor U0126 and a selective p38 inhibitor SB203580, indicating that ERK1/2 and p38 activities were essential for the induction of MMP-1, MMP-13 and TIMP-1 expression. Furthermore, type II collagen expression was increased by U0126 and SB203580. However, activation of the JNK pathway was not essential for enhancement of MMP-1, MMP-13 and TIMP-1 expression in chondrosarcoma. The results indicated that inhibition of the activity of distinct MAPKs may serve as a potent method to inhibit MMPs and increase type II collagen expression in chondrosarcoma.

The present study demonstrated that expression of MMP-1 and MMP-13 is dependent on the activity of MAPK pathways in the succession of chondrosarcoma invasion and metastasis, and has provided the theoretical basis for chondrosarcoma therapy with MAPK inhibitors. The identification of targets to inhibit ECM degradation by MMPs will aid in the treatment of chondrosarcoma. 


\section{Acknowledgements}

The authors would like to thank Dr Si-yuan Liu (The Third Hospital of Hebei Medical University, Shijizhuang, China) for collecting the pathological specimens.

\section{References}

1. Leddy LR and Holmes RE: Chondrosarcoma of bone. Cancer Treat Res 162: 117-130, 2014.

2. Gelderblom H, Hogendoorn PC, Dijkstra SD, van Rijswijk CS, Krol AD, Taminiau AH and Bovée JV: The clinical approach towards chondrosarcoma. Oncologist 13: 320-329, 2008.

3. Van Gompel JJ and Janus JR: Chordoma and chondrosarcoma. Otolaryngol Clin North Am 48: 501-514, 2015.

4. Yuan J, Dutton CM and Scully SP: RNAi mediated MMP-1 silencing inhibits human chondrosarcoma invasion. J Orthop Res 32: 1467-1474, 2005

5. Sakimura R, Tanaka K, Yamamoto S, Matsunobu T, Li X, Hanada M, Okada T, Nakamura T, Li Y and Iwamoto Y: The effects of histone deacetylase inhibitors on the induction of differentiation in chondrosarcoma cells. Clin Cancer Res 13 275-282, 2007.

6. Taniwaki K, Fukamachi H, Komori K, Ohtake Y, Nonaka T, Sakamoto T, Shiomi T, Okada Y, Itoh T, Itohara S, et al: Stroma-derived matrix metalloproteinase (MMP)-2 promotes membrane type 1-MMP-dependent tumor growth in mice. Cancer Res 67: 4311-4319, 2007.

7. Park JM, Kim A, Oh JH and Chung AS: Methylseleninic acid inhibits PMA-stimulated pro-MMP-2 activation mediated by MT1-MMP expression and further tumor invasion through suppression of NF-kappaB activation. Carcinogenesis 28: 837-847, 2007.

8. Chiu YC, Yang RS, Hsieh KH, Fong YC, Way TD, Lee TS, Wu HC, Fu WM and Tang CH: Stromal cell-derived factor-1 induces matrix metalloprotease-13 expression in human chondrocytes. Mol Pharmacol 72: 695-703, 2007.

9. Brown GT and Murray GI: Current mechanistic insights into the roles of matrix metalloproteinases in tumour invasion and metastasis. J Pathol 237: 273-281, 2015.

10. Moon JW, Choi JH, Lee SK, Lee YW, Lee JO, Kim N, Lee HJ Seo JS, Kim J, Kim HS, et al: Promoter hypermethylation of membrane type 3 matrix metalloproteinase is associated with cell migration in colorectal adenocarcinoma. Cancer Genet 208: 261-270, 2015

11. Zhang X, Yin P, DI D, Luo G, Zheng L, Wei J, Zhang J, Shi Y, Zhang $\mathrm{J}$ and $\mathrm{Xu}$ N: IL- 6 regulates MMP-10 expression via JAK2/STAT3 signaling pathway in a human lung adenocarcinoma cell line. Anticancer Res 29: 4497-4501, 2009.

12. Galoian KA, Garamszegi N, Garamszegi SP and Scully SP Molecular mechanism of tenascin-C action on matrix metalloproteinase-1 invasive potential. Exp Biol Med (Maywood) 232: 515-522, 2007.

13. Pritchard AL and Hayward NK: Molecular pathways: Mitogenactivated protein kinase pathway mutations and drug resistance. Clin Cancer Res 19: 2301-2309, 2013.

14. Klein AM, Zaganjor E and Cobb MH: Chromatin-tethered MAPKs. Curr Opin Cell Biol 25: 272-277, 2013.

15. Chen PN, Hsieh YS, Chiou HL and Chu SC: Silibinin inhibits cell invasion through inactivation of both PI3K-Akt and MAPK signaling pathways. Chem Biol Interact 156: 141-150, 2005

16. Ozen E, Gozukizil A, Erdal E, Uren A, Bottaro DP and Atabey N: Heparin inhibits Hepatocyte Growth Factor induced motility and invasion of hepatocellular carcinoma cells through early growth response protein 1. PLoS One 7: e42717, 2012.

17. Lin CW, Hou WC, Shen SC, Juan SH, Ko CH, Wang LM and Chen YC: Quercetin inhibition of tumor invasion via suppressing PKC/ERK/AP-1-dependent matrix metalloproteinase-9 activation in breast carcinoma cells. Carcinogenesis 29: 1807-1815 2008.

18. Zhao HJ, Liu T, Mao X, Han SX, Liang RX, Hui LQ, Cao CY, You Y and Zhang LZ: Fructus phyllanthi tannin fraction induces apoptosis and inhibits migration and invasion of human lung squamous carcinoma cells in vitro via MAPK/MMP pathways. Acta Pharmacol Sin 36: 758-768, 2015.
19. Cohen M, Meisser A, Haenggeli L and Bischof P: Involvement of MAPK pathway in TNF-alpha-induced MMP-9 expression in human trophoblastic cells. Mol Hum Reprod 12: 225-232, 2006.

20. Livak KJ and Schmittgen TD: Analysis of relative gene expression data using real-time quantitative pcr and the 2(-Delta Delta C(T)) method. Methods 25: 402-408, 2001.

21. Iwamoto Y: Diagnosis and treatment of Ewing's sarcoma. Jpn J Clin Oncol 37: 79-89, 2007.

22. Tan TW, Yang WH, Lin YT, Hsu SF, Li TM, Kao ST, Chen WC, Fong YC and Tang CH: Cyr61 increases migration and MMP-13 expression via alphavbeta3 integrin, FAK, ERK and AP-1-dependent pathway in human chondrosarcoma cells. Carcinogenesis 30: 258-268, 2009.

23. Tarín C, Gomez M, Calvo E, López JA and Zaragoza C: Endothelial nitric oxide deficiency reduces MMP-13-mediated cleavage of ICAM-1 in vascular endothelium: A role in atherosclerosis. Arterioscler Thromb Vasc Biol 29: 27-32, 2009.

24. Daniele A, Zito AF, Giannelli G, Divella R, Asselti M, Mazzocca A, Paradiso A and Quaranta M: Expression of metalloproteinases MMP-2 and MMP-9 in sentinel lymph node and serum of patients with metastatic and non-metastatic breast cancer. Anticancer Res 30: 3521-3527, 2010.

25. Määtta M, Talvensaari-Mattila A, Turpeenniemi-Hujanen T and Santala M: Matrix metalloproteinase-2 (MMP-2) and -9 (MMP-9) and their tissue inhibitors (TIMP-1 and TIMP-2) in differential diagnosis between low malignant potential (LMP) and malignant ovarian tumours. Anticancer Res 27: 2753-2758, 2007.

26. Roomi MW, Kalinovsky T, Niedzwiecki A and Rath M: Modulation of uPA, MMPs and their inhibitors by a novel nutrient mixture in human colorectal, pancreatic and hepatic carcinoma cell lines. Int J Oncol 47: 370-376, 2015.

27. Kirimlioğlu H, Türkmen I, Bassüllüi N, Dirican A, Karadağ N and Kirimlioğlu V: The expression of matrix metalloproteinases in intrahepatic cholangiocarcinoma, hilar (Klatskin tumor), middle and distal extrahepatic cholangiocarcinoma, gallbladder cancer, and ampullary carcinoma: Role of matrix metalloproteinases in tumor progression and prognosis. Turk J Gastroenterol 20: 41-47, 2009.

28. Tang CH, Tan TW, Fu WM and Yang RS: Involvement of matrix metalloproteinase-9 in stromal cell-derived factor-1/CXCR4 pathway of lung cancer metastasis. Carcinogenesis 29: 35-43, 2008.

29. Safranek J, Pesta M, Holubec L, Kulda V, Dreslerova J, Vrzalova J, Topolcan O, Pesek M, Finek J and Treska V: Expression of MMP-7, MMP-9, TIMP-1 and TIMP-2 mRNA in lung tissue of patients with non-small cell lung cancer (NSCLC) and benign pulmonary disease. Anticancer Res 29: 2513-2517, 2009.

30. Yamada T, Oshima T, Yoshihara K, Tamura S, Kanazawa A, Inagaki D, Yamamoto N, Sato T, Fujii S, Numata K, et al: Overexpression of MMP-13 gene in colorectal cancer with liver metastasis. Anticancer Res 30: 2693-2699, 2010.

31. Vogel C, Chan A, Gril B, Kim SB, Kurebayashi J, Liu L, Lu YS and Moon H: Management of ErbB2-positive breast cancer: Insights from preclinical and clinical studies with lapatinib. Jpn J Clin Oncol 40: 999-1013, 2010.

32. Ellington AA, Berhow MA and Singletary KW: Inhibition of Akt signaling and enhanced ERK1/2 activity are involved in induction of macroautophagy by triterpenoid B-group soyasaponins in colon cancer cells. Carcinogenesis 27: 298-306, 2006

33. Wollmann W, Goodman ML, Bhat-Nakshatri P, Kishimoto H, Goulet RJ Jr, Mehrotra S, Morimiya A, Badve S and Nakshatri H: The macrophage inhibitory cytokine integrates AKT/PKB and MAP kinase signaling pathways in breast cancer cells. Carcinogenesis 26: 900-907, 2005.

34. Wu K, Xu L, Zhang L, Lin Z and Hou J: High jagged1 expression predicts poor outcome in clear cell renal cell carcinoma. Jpn J Clin Oncol 41: 411-416, 2011.

35. de Seranno S and Meuwissen R: Progress and applications of mouse models for human lung cancer. Eur Respir J 35: 426-443, 2010.

36. Chen L, Mayer JA, Krisko TI, Speers CW, Wang T, Hilsenbeck SG and Brown PH: Inhibition of the p38 kinase suppresses the proliferation of human ER-negative breast cancer cells. Cancer Res 69: 8853-8861, 2009.

37. Miao Y, Zhang Y, Wan H, Chen L and Wang F: GABA-receptor agonist, propofol inhibits invasion of colon carcinoma cells. Biomed Pharmacother 64: 583-588, 2010. 